Ann. Zootech., I975, 24 (2), 209-215.

\title{
LE TOURTEAU DE COLZA DANS L'ALIMENTATION ANIMALE. UTILISATION COMPARÉE DES TOURTEAUX DE COLZA TOASTÉ, DE COLZA FERMENTÉ, DE SOJA ET D'ARACHIDE POUR L'ENGRAISSEMENT DES JEUNES TAUREAUX
}

\author{
Y. GEAY et C. BERANGER \\ avec la collaboration technique de Robert Jailuer, Roland Jailler et G. Cuylle \\ Laboratoire de la Production de Viande, \\ Centre de Recherches de Clermont-Ferrand, I. N.R. A., \\ Theix, Sains Genès Champanelle, 63110 Beaumont
}

\section{RÉ,SUMÉ}

Nous avons comparé la croissance, la composition des carcasses et le fonctionnement de la thyroïde de 64 jeunes bovins mâles à l'engrais, recevant l'un des quatre tourteaux suivants : colza toasté, colza fermenté (STARoN, I970), soja et arachide distribués en association avec du maïs grain pour complémenter une ration d'ensilage de blé puis de pulpes déshydratées.

Les proportions respectives de maïs et de tourteau étaient telles que le mélange devait fournir aux animaux une quantité identique de matières azotées totales et d'énergie.

Le tourteau de colza toasté n'a pas permis aux animaux de réaliser des gains de poids vif ( I I $99 \pm$ I $3 \mathrm{I} \mathrm{g} / \mathrm{j}$ ) identiques à ceux obtenus avec les tourteaux d'arachide (I $266 \pm \mathrm{I} 3 \mathrm{I} \mathrm{g} / \mathrm{j}$ ) ou de soja ( 1 3OI \pm 242$)$ mais il a été utilisé presqu'aussi efficacement $(5,47 \mathrm{UF} / \mathrm{kg}$ gain contre 5,42 et 5,37 respectivement pour l'arachide et le soja). L'ingestion du tourteau de colza toasté a entrainé une augmentation significative $(\mathrm{P}<0,00 \mathrm{I})$ du poids des thyroïdes $(74, \mathrm{I}$ g contre 32,4 et 36,9 respectivement pour les animaux ayant reçu le soja ou l'arachide), mais n'a pas modifié les proportions de muscles et de gras dans les carcasses.

Le tourteau de colza fermenté n'a pas, comme le précédent, entrainé une diminution des quantités de matière sèche ingérées du reste de la ration. Les animaux qui en recevaient ont même ingéré plus d'aliments que ceux des régimes "soja " ou "arachide", avec une efficacité alimentairc très voisine. La fermentation n'a toutefois pas éliminé totalement les substances à activité goitrigène : celles-ci ont accru significativement $(\mathrm{P}<0,05)$, par rapport au régime "soja ", le poids des glandes thyroïdes $(56,8 \mathrm{~g}$ contre 32,4$)$.

\section{INTRODUCTION}

Le colza contient des thioglucosides qui, par hydrolyse enzymatique, donnent naissance à des substances qui diminuent son appétibilité (isothiocyanates) ou ont une activité goitrigène (L-5-viny1-2-oxazolidinéthione : VTO) (cf. Revues de JAR- 
RIGE, I970 ; ZELTER, I970). Cette action antithyrö̈dienne du VTO a été constatée chez le Rat (Kennedy et Purves, I94I), le Porc (Nordfeld et al., I954; Hussar et Bowland, I959), les volailles (TURNER, I946 ; CIANDinin et BAILY, I960 ; CALET, I970) et, aussi chez les brebis gestantes ('THÉRIEZ et al., I97I). Certaines variétés de soja peuvent avoir également une action goitrigène chez le ruminant: HEMkEN et al. (I97I) ont en effet constaté que du tourteau de soja distribué à des vaches laitières gestantes pouvait provoquer une hypertrophie de la glande thyroïde des veatux à leur naissance.

L'hypofonctionnement de la thyroïde, consécutif à l'ingestion d'antithyroïdiens, se traduit en général, par une augmentation de la rétention d'eau, comme l'ont observé Muhrer et al. (I947), Winchester et Andrews (I953) chez le Porc, et une diminution de l'adipogénèse (MuHRER et al., I947 ; WINCHESTER et ANDREWS, I953; Pfarson et al., I966 chez le Porc ; CaL,ET, i97o chez le Poulet). Terril, ct al. (I950) ont même constaté chez le Porc une augmentation de la rétention azotée, mais elle fut de courte durée. Ces modifications de la composition corporelle pourraient présenter un intérêt zootechnique pour la production de carcasses lourdes, à partir de taurillons de races précoces, dans la mesure où elles permettraient de ralentir chez ces animaux la formation trop rapide des dépôts adipeux.

Pour atténuer ou supprimer l'action des substances goitrigènes et éliminer les isothiocyanates qui limitent l'appétibilité du tourteau de colza, un certain nombre de traitements ont été proposés (cf. Revue de ZELTER, I970). BÉRANGER et GRENET (I969), GRENET et Journet (I97I), ont montré que le "toastage " (grillage suivi d'injection de vapeur dans la masse) permettait d'améliorer sensiblement l'appétibilité du tourteau de colza chez les bovins, en éliminant une partie des isothiocyanates. STARON (I970) a mis au point une méthode permettant d'éliminer les V'TO, par fermentation du tourteau de colza en milieu aqueux en présence du champignon Geotrichum candidum.

Afin d'étudier l'intérêt respectif de ces différents aliments dans l'alimentation des jeunes bovins, nous avons comparé la croissance, la composition des carcasses et le fonctionnement de la thyroïde de jeunes bovins mâles non castrés à l'engrais recevant 1'un des quatre tourteaux suivants : colza toasté, colza fermenté (procédé Staron), soja et arachide (témoin).

\section{MATÉRIEL ET MÉTHODES}

Pour comparer les quatre tourteaux précédents, nous les avons clistribués en mélange avec du maïs grain condensé en complément d'une ration constituée successivement d'ensilage de blé immature ( 49 jours) et de pulpes de betteraves déshydratées (ro5 jours). Les proportions respectives de tourteaux et de maïs ont été calculées de façon que les quantités d'énergie (UF) et de matières azotées totales apportées par ce mélange, soient identiques dans chacun des régimes, l'aliment de base (ensilage de blé puis pulpes de betteraves déshydratées) étant distribué ad libitum. Le tableau I précise la composition des aliments utilisés. La valeur énergétique de l'ensilage de blé a été estimée, compte tenu de sa teneur en cellulose, à partir des résultats obtenus par C. Demarquilly et J. Andrieu sur i i ensilages de blé ; celle des autres aliments a été estimée, compte tenu de leur composition, à partir du manuel pour le calcul de la valeur nutritive des fourrages édité par Ie Laboratoire d'Oosterbeek aux Pays-Bas.

Chacun des quatre tourteaux a été distribué à un lot de 16 taurillons Salers âgés de 9 mois et pesant $319 \mathrm{~kg}$ en moyenne. Les animaux placés en stabulation libre sous un hangar ouvert, 
ont été abattus par bloc de 4 (un animal de chaque régime) lorsque l'état d'engraissement du taurillon recevant le colza toasté a été jugé satisfaisant, en moyenne après i 54 jours d'expérience $(\sigma=25)$.

TABI,EAU I

Composition de la matière sèche des aliments utilisés

(P. IOO)

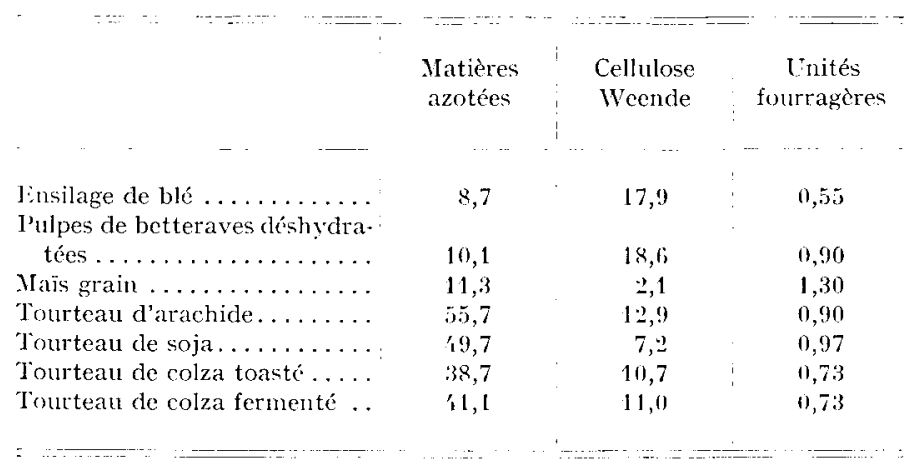

Les mesures suivantes ont été effectuées : pesée des animaux, ì intervalles de deux semaines, double pesée (pesée deux jours consécutifs à la même heure) tous les mois et avant le départ pour l'abattoir, pesée des quantités distribuées et refusécs de chaque aliment par lot et par jour, pesée du corps vide, de la carcasse et de la glande thyroïle. Un échantillon de chaque glande a été fixé dans une solution de Boin-Holland sublimé, a fin cl'être examiné au microscope. Par ailleurs, au début, au milieu et à la fin de la période cl'engraissement, on a prélevé du sang jugulaire sur 8 animaux de chaque lot pour mesurer la teneur en thyroxine $\left(T_{4}\right)$ et triodothyronine $\left(T_{3}\right)$ circulantes. On a mesuré le taux de précurseurs hormonaux au niveau des thvroïles de ces 8 animaux (1).

\section{RÉSULTATS ETT DISCUSSION}

a) Les animaux recevant les régimes comportant du colza n'ont pas consommé, au total, autant d'azote que ceux recevant du soja ou de l'arachide. Cela provient de quelques "refus " de tourteaux, dus soit à une trop grande dureté des granulés de tourteau de colza fermenté, soit à une appétibilité faible du tourteau de colza toasté, phénomène déjà observé par BÉRANGER et GRENET (I969), ThÉrRIEZ et al., (I97I). Les taurillons recevant le régime comportant le tourteau de colza toasté ont consommé également moins d'énergie que les autres, bien que les différences soient faibles. Ces quantités d'azote et d'énergie ingérées plus faibles se sont en outre accompagnées d'une très légère diminution de l'efficacité alimentaire par rapport à celle des autres régimes, particulièrement celle du régime "soja ». Ces résultats confirment ceux obtenus par JARRIGE (résultats non publiés) comparant le tourteau de colza au tourteau de lin. En outre, le poids vif vide et le poids des carcasses des animaux recevant le colza toasté ont été significativement inférieurs à ceux des taurillons recevant le soja $(\mathrm{P}<0,05)$. En revanche, la composition des carcasses n'a pas

(1) Les dosayes des précurseurs hormonaux et des hormones circulantes on tété réalisés par Mme MicheL.oT du Laboratoire d'Études métaboliques des Molécules marquées de l'I. N. S. E. R. M. à ClermontFerrand et feront l'objet d'une publication ultérieure. 
été significativement différente d'un régime à l'autre. Le colza n'a donc pas diminué l'état d'engraissement des taurillons. Ceci rejoint les observations de THÉriEz et al. (I97I) sur agneaux et de BowlAND (I972) sur porcs à l'engrais. Il faut cependant remarquer que la proportion de gras (I0,3 à Io,8 p. IOo) dans la carcasse était particulièrement faible pour des taurillons de race Salers. Nous avons en effet obtenu une valeur moyenne de 12,6 p. Ioo pour 236 animaux de la même race, abattus à des poids de carcasse voisins. Les antithyroïdiens du tourteau de colza n'ont pas non plus accru la rétention d'eau comme l'ont observé chez le porc Munrer et al. (I947), WinchesTER et ANDREws (r953) à l'aide d'antithyroïdiens de synthèse.

\section{TABLEAU 2}

Ltilisation de différents tourteaux par les jeunes bovins (moyenne \pm écart-type)

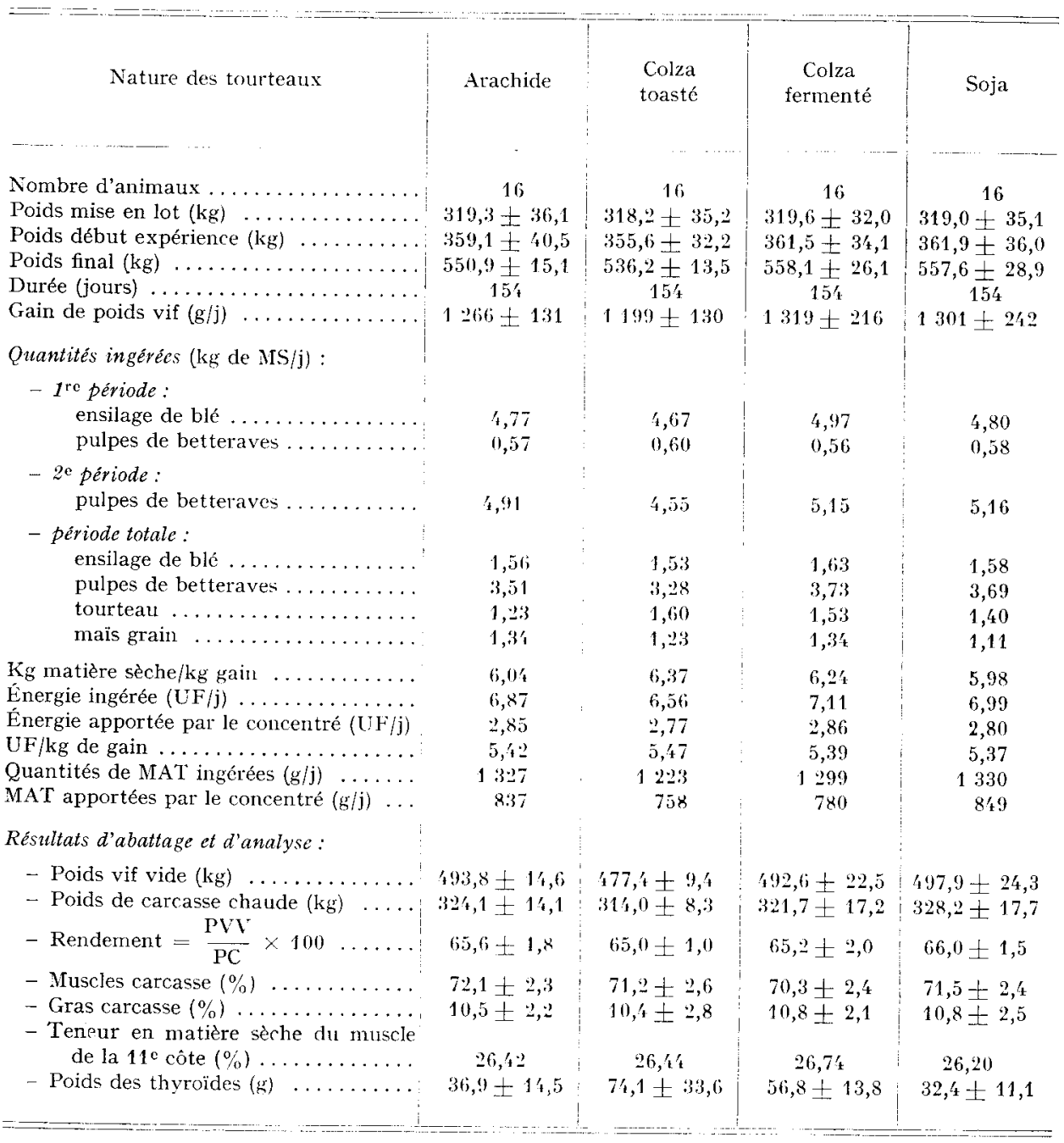


Le principal objectif de cette expérience qui était d'étudier l'intérêt du tourteau de colza pour la production de carcasses maigres, nous a conduit à distribuer des quantités importantes de tourteau, et par suite, des rations ayant un taux azoté très élevé (I6 à I 7 p. Ioo de matières azotées totales $/ \mathrm{kg}$ de matière sèche). Les différences de performances constatées entre les animaux recevant le colza toasté et ceux recevant le soja notamment, ont, de ce fait, pu être accrues et n'auraient peut-être pas été sensibles si les quantités de tourteau distribuées avaient été limitées au niveau optimum (775 g de tourteau de soja, I $\mathrm{kg}$ de tourteau de colza toasté).

b) Conformément au protocole, les animaux recevant le colza toasté ou fermenté, ont ingéré les mêmes quantités d'énergie et d'azote provenant du mélange " tourteaumaîs ". Cependant, le colza fermenté semble avoir stimulé l'appétit des animaux, puisque ceux-ci ont consommé, par rapport à leurs homologues du régime " colza toasté ", une quantité supérieure de matière sèche d'ensilage (II p. IOO) et surtout de pulpes déshydratées (I3,7 p. IOO). Cette ingestion supérieure de matière sèche et donc d'énergie et d'azote, s'est traduite par un gain de poids vif et un poids de carcasse plus élevés (mais non significativement cependant), respectivement de 6,0 et de 2,4 p. Ioo, accompagnés d'une amélioration de l'efficacité alimentaire qui la situe au niveau de celle du régime " soja ". En revanche, la composition des carcasses, estimée à partir de celle de la I I ${ }^{\mathrm{e}}$ côte (GeAy et BÉRANGre, I969) a été identique d'un régime à l'autre.

Les animaux recevant ce régime " colza fermenté " ont réalisé le même croît journalier et fourni des carcasses de même poids et de même composition que ceux recevant le régime "soja ». Ils ont cependant ingéré moins d'azote et plus d'énergie. Il. apparait donc que la valeur énergétique du tourteau de colza fermenté, adoptée dans nos calculs $(0,73 \mathrm{UF} / \mathrm{kg}$ de matière sèche), est vraisemblablement excessive par rapport à la valeur réelle (laquelle doit être proche de $0,65 \mathrm{UF}$ ). Ceci rejoint les résultats obtenus par Guir.aume (Communication personnelle) chez le Poulet.

c) A l'abattage, les glandes thyroïdes des animaux ayant consommé l'arachide et le soja étaient significativement moins lourdes $(\mathrm{P}<\mathrm{o,00I})$ que celles des animaux ayant reçu le colza toasté, celles des animaux au colza fermenté ayant un poids intermédiaire. Ainsi, contrairement aux observations de VIRTANEN (I959), les ruminants apparaissent sensibles, comme le Porc, aux goitrigènes du colza qui ne sont pas entièrement dégradés dans le rumen. Dans cette expérience, l'hypertrophie de la thyroïde qui en est résultée, s'est accompagnée d'une hyposécrétion de thyroxine $\left(\mathrm{T}_{1}\right)$ et de triodothyronine $\left(\mathrm{T}_{3}\right)$, ainsi que d'une modification du rapport des concentrations en précurseurs hormonaux MI'T/DI'T comme l'ont observé 'THÉRIEZ et al. (I97I) sur agneaux. Ces modifications ont été apparentes aussi bien chez les animaux ayant reçu le colza toasté que chez ceux recevant le colza fermenté.

Ainsi, bien que l'analyse ait révélé que la fermentation avait éliminé les VTO et les isothiocyanates du colza, certaines substances à action goitrigène restent présentes.

En conclusion, le régime “ colza toasté n'a pas permis aux animaux de réaliser des gains de poids vif identiques à ceux obtenus avec les régimes " arachide " ou " soja ", mais il a été utilisé presqu'aussi efficacement. Cependant, les antithyroïdiens du tourteau de colza ne semblent pas présenter un intérêt zootechnique important au niveau de la maîtrise de la composition corporelle des jeunes bovins. 
Le tourteau de colza fermenté, utilisé lors de cette expérience, insuffisamment consommé pour des raisons technologiques, n'a cependant pas entrainé, comme le tourteau précédent, une consommation plus faible du reste de la ration. Il a également permis une légère amélioration de l'efficacité alimentaire sans modification de la composition de la carcasse.

Enfin, si certaines variétés de soja, comme l'ont montré HEMkEN et al. (I97I), peuvent avoir une action goitrigène, celle-ci ne s'est pas manifestée avec le tourteau que nous avons utilisé. Le régime "soja " a même permis aux animaux de réaliser des performances très légèrement supérieures à celles réalisées avec le régime " arachide ).

\title{
Requ pour publication en décembre 1974.
}

\section{SUMMARY}

\author{
RAPESEED OII, MEAL, IN ANIMAL FEEDING. \\ COMPARATIVE, U'TILIZATION OF THE, FOLLOWING OIL MEALS : \\ TOASTED RAPESEED, FERMENTED RAPESEED, \\ SOYBEAN AND PEANUT FOR THE FATTENING OF YOUNG BULLS
}

In the present study, comparison was made on growth, carcass composition and thyroid function of 64 fattening young bulls receiving one of the four following oil meals : toasted rapeseed, fermented rapeseed (STARos, I970), soybean and peanut offered together with maize grain to supplement the basal diet composed first of wheat silage and then of dried beet pulps.

The proportions of maize and oil meal were calculated in order to provide identical amounts of total crude protein and energy to the animals.

The live weight gains obtained with toasted rapeseed oil meal ( I I99 \pm I3 1 g/day) were lower than those obtained with peanut oil meal (I $266 \pm$ I $3 \mathrm{I}$ g/day) or soybean oil meal (I $30 \mathrm{r} \pm 242$ ), but the feed conversion ratio was almost the same $(5.47 \mathrm{UF} / \mathrm{kg}$ weight gain versus 5.42 and 5.37 respectively for peanut and soybean oil meal). The intake of toasted rapeseed oil meal led to a significant increase $(\mathrm{P}<0.00 \mathrm{I})$ in the wejght of the thyroid glands (74.I $\mathrm{g}$ versus 32.4 and $36.9 \mathrm{~g}$ respectively for the animals fed with soybean or peanut oil meal), but did not modify the proportions of lean and fat in the carcasses.

Fermented rapeseed oil meal did not, as the previous one did, cause any decrease in the intake of dry matter in the rest of the diet. The animals fed with this oil meal even exhibited a higher intake level than those receiving the "soybean" and "peanut" diets, and very similar feed efficiency. However, the fermentation did not entirely eliminate the goitrogenic substances. As compared to the "soybean" diet, these substances significantly increased $(\mathrm{P}<0.05)$ the weight of the thyroid glands $(56.8$ versus 32.4$)$.

\section{RÉFÉRENCES BIBLIOGRAPHIQUES}

Béranger C., Grenet N., i 969 . Le tourteau de colza dans l'alimentation animale. I. Influence de divers procédés de fabrication sur les quantités ingérées par des bovins en croissance. Ann. Zootech., 18, 239-247.

BoWland J.-P., I972. Unprocessed rapeseed treated with propionic acid in diets of growing pigs : performance, energy and protein digestibility, and nitrogen retention, carcass measurement, and fatty acid composition of backfat. Can. J. Anim. Sci, 52, 553-562.

CALET L., I970. Compte rendu des Journées Internationales sur le colza., 55 I-559.

Clandinin D. R., Baily L., r96o. Rapeseed oil meal studies. II. Effects of feeding rapeseed oil meal on the structure of the thyroid glands of chickens. Poult. Sci., 39, I 239 (Abstr.). 
Geay Y., Béranger C., rg69. Estimation de la composition de la carcasse des jeunes bovins à partir de la composition d'un morceau monocostal au niveau de la I I cóte. Ann. Zootech., 18, 65-77.

Grenet N., Journet M., I97i. Le tourteau de colza dans l'alimentation animale. III. Influence du procédé de fabrication et de la proportion de tourteau dans l'aliment concentré sur les quantités ingérées par les vaches laitieres et sur la production et la composition du lait. Ann. Zootech., 20, 437-449.

Hemken R. W., Vandersall J. H., Sass B. A., Hibis J. W., 197x. Goitrogenic effects of a corn silage-soybean meal supplemented ration. J. Dairy Sci., 54, 85-88.

Hussar N., Bowland J.-P., r959. Rapeseed oil meal as a protein supplement for swine and rats. I. Rate of gain, efficiency of food utilization, carcass characteristics and thyroid activity. Can.J.Anim. Sci., 39, 84-93.

JARrige R., I970. Utilisation des tourteaux de colza dans l'alimentation des animaux domestiques. Compte rendu des Journées Internationales sur le colza., 529-554.

Kennedy T. H., Purves H. D., I94I. Studies on experimental goitre. I. The effect of Brassica seed diets on rats. Brit. J. Exp. Path., 22, $24 \mathrm{I}-244$.

Muhrer M. E., Warner D. R., Palmer Z., Hogan A. G., 1947. Effect of thiouracil and protamone on growing swine. J. Anim. Sci., 6, $489-490$.

Nordfeldt S., Gellersted N., Falkmer S., 1954. Studies of rapeseed meal and its goitrogenic effect in pigs. A nutritional and histopathological study. Acta. Pathol. Microbiol. Scand., 35, 2 I7-236.

Pearson A. M., Reineke E. P., Hoefer J. A., Morrow R. E., rg66. Effect of environmental temperature, and thiouracyl feeding upon growing-fattening pigs. J. Anim. Sci., 26, 994-999.

Staron T., r97o. Une méthode de détoxification des tourteaux de colza par voie biologique. Compte rendu des Journées Internationales sur le colza. Paris 26-30 mai r970, 482-496.

Terril S. W., Hamilton T. S., Krider J. L., Carroll W. E., i95o. Carcass composition and nitrogen balances of swine fed thiouracil. J. Anim. Sci., 9, 58.

Thériez M., Grenet N., Molénat G., I97I. Le tourteal de colza dans l'alimentation animale. IV. Étucle comparée de l'appétibilité et de la valeur alimentaire des tourteaux de colza et de lin pour l'agneau à l'engraissement et la brebis gestante; effets sur la glande thyroïde. Ann. Zootech., 20, 45I-463.

Turner C. W., 1946. Effect of rapeseed on the thyroid of the chick. Poult Sci., 25, i 86-187.

VirTanen A. I., I959. On the transfer of L-5-vinyl-2-thisoxazolidone from the rumen to the milk. Acta. Chem. Scand., 13, 1043 (Abstr.).

Winchester C. F., Andrews F. N., 1953. Some uses of drugs and hormones in beef cattle sheep, and swine husbandry. National Academy of Sciences. National Research Council. Pub., 256, 31-40.

ZeLter S. Z., I970. Quelques aspects nutritionnels de la qualité des tourteaux de colza et de navette. Compte rendu des Journées Internationales sur le colza, 41 I-4.39. 